\title{
Urinary angiotensinogen as a marker of intrarenal angiotensin II activity in adolescents with primary hypertension
}

\author{
Elżbieta Kuroczycka-Saniutycz • Anna Wasilewska • \\ Agnieszka Sulik • Robert Milewski
}

Received: 25 October 2012 /Revised: 19 February 2013 / Accepted: 20 February 2013 / Published online: 26 March 2013

(C) The Author(s) 2013. This article is published with open access at Springerlink.com

\begin{abstract}
Background Experimental and epidemiological studies have demonstrated that urinary angiotensinogen (AGT) is a novel biomarker for the intrarenal activity of the reninangiotensin system in hypertension (HT). Several largescale epidemiological studies have shown that an elevated serum uric acid (SUA) level is associated with HT. The aim of our study was to assess urinary AGT excretion and its correlation with SUA level, the lipid profile, and the body mass index (BMI) Z-score in hypertensive adolescents. Methods Participants were divided into two groups: (1) the group with confirmed HT consisting of 55 subjects with primary HT and (2) the reference (R) group consisting of 33 subjects with white-coat HT. A commercial enzymelinked immunosorbent assay (ELISA) kit was used to determine urinary AGT concentration.

Results The urinary AGT/creatinine (cr.) ratio in subjects in the HT group was significantly higher than that in the reference group $(p<0.01)$ and showed a strong positive correlation with SUA $(r=0.47, p<0.01)$. The relationship between the AGT/cr. ratio and SUA levels after controlling for age, gender and BMI Z-score continued to show a significant association.
\end{abstract}

E. Kuroczycka-Saniutycz $\cdot$ A. Wasilewska $(\bowtie)$

Department of Pediatrics and Nephrology,

Medical University of Białystok, ul. Waszyngtona 17,

15-274 Białystok, Poland

e-mail: annwasil@interia.pl

\section{A. Sulik}

Department of Rheumatology and Internal Medicine,

Medical University of Bialystok, Białystok, Poland

R. Milewski

Department of Statistics and Medical Informatics,

Medical University of Bialystok, Białystok, Poland
Conclusions The most obvious finding to emerge from this study is that in adolescents with primary HT, the increased urinary excretion of AGT correlated with hyperuricemia, although large, multicenter studies are needed to confirm this observation.

Keywords Adolescents · Urinary angiotensinogen · Hyperuricemia · Hypertension

\section{Introduction}

Epidemiological and experimental data suggest that uric acid could play a contributory role in the pathogenesis of elevated blood pressure [1-4]. Feig et al. [5] reported that an increased uric acid level of $>5.5 \mathrm{mg} / \mathrm{dL}$ in $90 \%$ of adolescents with newly diagnosed primary hypertension (HT). Saito et al. [6] confirmed that the renin-angiotensin system (RAS) is also strongly related to elevated serum uric acid (SUA) levels in hypertensive subjects. These authors found that uric acid stimulated proliferation, angiotensin II production and oxidative stress in vascular smooth muscle cells (VSMCs) through the tissue RAS.

In recent years, the role of the intrarenal RAS in the pathophysiology of both HT and renal injury has become a focus of interest to researchers. Considerable attention has been paid to the significance of the local/tissue RAS in different tissues, including the brain, heart, adrenal glands, vasculature and kidneys [7-13]. Urinary angiotensin II is unstable and, therefore, cannot be used as a reliable marker of intrarenal RAS activity in clinical studies. Experimental studies have demonstrated that angiotensinogen (AGT) levels in renal tissues reflect the activity of the intrarenal RAS [12-15].

Katsurada et al. [16] recently developed a sandwich enzyme-linked immunosorbent assay (ELISA) system to directly measure urinary human AGT levels. Urinary AGT 
levels have been shown to be highly correlated with intrarenal AGT and angiotensin II levels. Consequently, urinary AGT concentration has been suggested as a reliable marker for intrarenal RAS activity [17-19].

The aim of this study was to assess urinary AGT excretion in hypertensive adolescents and to evaluate its correlation with SUA, the lipid profile and body mass index (BMI) $Z$-score.

\section{Patients and methods}

This was a prospective cohort study of hypertensive adolescents. The study included 88 subjects ( 33 girls, 55 boys) aged 11-18 years who were referred to our unit (Department of Pediatrics and Nephrology, The Medical University of Białystok, Poland) between May 2010 and September 2011 to confirm or rule out the diagnosis of HT. The majority of patients were referred by general practitioners who had identified elevated blood pressure (BP) in the patient. We divided the adolescents into two groups based on ambulatory blood pressure monitoring (ABPM): (1) a hypertensive group (HT group) consisting of patients with confirmed primary HT; (2) a reference (R) group consisting of patients with white-coat HT. In addition, a group of adolescents with HT was subsequently divided into two subgroups: (1) HT $\mathrm{HU}(+)$ consisting of patients with HT accompanied by hyperuricemia; (2) HT HU(-), i.e. subjects with HT and normal SUA levels.

The study group (HT) consisted of 55 hypertensive subjects [ 43 with hyperuricemia (SUA level of $\geq 5.5 \mathrm{mg} / \mathrm{dL}$ ) and 12 with a SUA level of $<5.5 \mathrm{mg} / \mathrm{dL}$ ].

Inclusion criteria were an age of 11-18 years, primary arterial HP [verified by ABPM as mean daytime and nighttime systolic BP (SBP) levels of $\geq 95$ th percentile for age, sex and height, and a load SBP or DBP of $>30 \%$ [20], normal clinical examination, renal ultrasound findings, normal electrocardiogram findings, normal levels of thyroid-stimulating hormone, and creatinine (cr.) and urinalysis within the normal range. Exclusion criteria were heart failure, diabetes mellitus, renal or hepatic dysfunction, hematological disease, systemic inflammatory conditions, autoimmune diseases, secondary forms of hypertension, girls treated with contraceptive pills, subjects treated with hypertensive agents and medications known to affect SUA levels and BP values.

The reference group consisted of 33 subjects in whom HT was excluded at the moment of examination on the basis of ABPM (mean daytime and nighttime SBP and DBP levels of $<90$ th percentile for age, sex and height and a load SBP and DBP of $<25 \%$ ). The participants were term-born, with normal birth weight, and were not receiving any medication at the time of the examination. The blood and urine test results of subjects in the reference group fell within the normal range. The family history of adolescents classified in this group did not reveal HT or other cardiovascular diseases. The medical history was negative for both diabetes and gout.

The protocol was approved by the Bioethics Committee of The Medical University of Białystok in accordance with the Declaration of Helsinki. Informed consent was obtained from parents of all participants and children older than 16 years of age.

For all subjects, careful clinical histories were taken and physical examinations were performed. Body weight and height were measured using a balance beam scale and pediatric wall-mounted stadiometer, and BMI was calculated as weight (in kilograms) divided by the square of height (meters squared). BMI $Z$-scores, reflecting the standard deviation score (SDS) for the age and gender appropriate BMI distribution, were calculated using the formula: BMI $Z$ scores $=(50$ percentile for age and gender $/ 1 / 2 \times(50$ percentile -3 percentile for age and gender).

After $12 \mathrm{~h}$ of overnight fasting, urine and blood samples were taken for the measurement of urinary AGT concentration and 24-h microalbuminuria, plasma renin activity, plasma aldosterone level, C-reactive protein, basal glucose level, lipid profile and serum creatinine, urea and SUA levels and morphology of peripheral blood. The urinary AGT concentration was measured using a commercially available ELISA kit (USCN Life Science Inc., Houston, TX) according to the manufacturer's instructions. In brief, a monoclonal antibody specific for AGT was used to detect AGT in the urine samples. Horseradish peroxidase-conjugated streptavidin was added, followed by a color-forming peroxidase substrate containing tetramethylbenzidine. The color was then measured at $450 \mathrm{~nm}$ by a microtiter plate reader and compared with a standard curve. Urinary AGT levels were expressed in nanograms per milliliter. Urinary creatinine concentration was used to normalize the AGT measurements to account for the influence of urinary dilution on its concentration. The urinary levels of creatinine were analyzed with Jaffé's method. The AGT levels were expressed as the urinary AGT/cr. ratio in nanograms per milligram creatinine. Plasma renin activity and plasma aldosterone concentrations were measured with commercially ELISA kits using monoclonal antibodies (R\&D Systems, Minneapolis, MN). Serum creatinine was determined by the Jaffé reaction, and uric acid was measured using a Hitachi apparatus (Hitachi, Chiyoda, Japan). The morphology of the peripheral blood were assessed on a Coulter analyzer MAXM (Beckman Coulter, Brea, CA). Serum cholesterol, high-density lipoprotein (HDL)-cholesterol and triglycerides were determined by the enzymatic method using a Hitachi model 912 apparatus. Serum glucose was measured with the Integra 800 analyzer (Roche Diagnostics, Mannheim, Germany). The 24-h urinary albumin excretion rate (UAER) was analyzed by a radioimmunoassay method. Albuminuria was considered as a 24-h UAER of $>30 \mathrm{mg} / 24 \mathrm{~h}$. Micro- and macroalbuminuria were defined as 24 -h UAER values of $30-300 \mathrm{mg} / 24 \mathrm{~h}$ and 
$>300 \mathrm{mg} / 24 \mathrm{~h}$, respectively. The estimated glomerular filtration rate (eGFR) was calculated from the Schwartz formula: eGFR $=\mathrm{k} \times \mathrm{G}(\mathrm{cm}) / \mathrm{Lcr} .(\mathrm{mg} / \mathrm{dL})$, where $\mathrm{k}$ is the agedependent coefficient $(0.55$ in boys aged $<12$ years and girls of any age, 0.70 in boys aged $>12$ years), $G$ is growth and Lcr. is the level of creatinine in the serum.

ABPM was performed using the SpaceLabs Medical oscillometric blood pressure monitor (SpaceLab, Redwood, WA). The monitors were programmed to measure BP every 15 min during daytime (800-2200 hours) and every $30 \mathrm{~min}$ during nighttime (2200-8.00 hours); however, the periods were corrected according to the subjects' diaries. Recording started between 8 and 9 a.m. and lasted for 24 h. Recordings with a minimum $80 \%$ of measurements and without breaks longer than $2 \mathrm{~h}$ were considered to be sufficient for analysis. The mean SBP and DBP were calculated separately for the 24-h period and for the awake and asleep periods. Also analyzed were the load systolic (LSBP) and diastolic (LDBP) BP during the day and night. HT on the basis of ABPM was defined as the mean daytime or nighttime SBP or DBP of $\geq 95$ th percentile and LSBP or LDBP daytime or nighttime levels of $>30 \%$ [20]. The values were adjusted by gender and body height according to the reference values provided by Wühl et al. [21]. Each subject or his parent was asked to record the bedtime and time of awakening. After $24 \mathrm{~h}$, the cuff and monitor were removed, and the data downloaded using the manufacturer's software.

\section{Statistical methods}

Data analysis was performed using the computer program Statistica 10.0 PL (StatSoft, Tulsa, IK). Discrete variables were expressed as counts (percentage), whereas continuous variables were expressed as the median and range, unless stated otherwise. The two groups were compared using the chi-square and Fisher exact tests for categorical variables and the $t$ test for continuous variables for normally distributed data and the Mann-Whitney test for the data distributed not normally. Multiple linear regression analyses were performed, including the AGT/cr ratio as dependent variable, with serum levels of total cholesterol, triglycerides, creatinine, uric acid, microalbuminuria, BMI $Z$-scores considered to be independent variables. Correlations between AGT/cr and other variables (clinical and laboratory parameters) were evaluated using standard methods, such as the Pearson's or Spearman's test accordingly. The value of $p<$ 0.05 was considered to be statistically significant.

\section{Results}

The demographic, clinical and ambulatory BP data for each group are summarized in Tables 1 and 2. The ABPM and laboratory results were successfully collected from 88 adolescents. Of these 88 subjects, 55 were HT and 33 had white-coat HT (reference group). The effect size between the hypertensive and reference groups was: $0.925326-$ power test $96 \%$. The median age did not differ between groups. Males were more frequently affected with HT than females, which is consistent with the available reports in the area [22]. In the group with HT, $46(83.6 \%)$ were males and nine $(16.4 \%)$ were females, whereas more girls $(n=24$, $72.7 \%)$ than boys $(n=9,27.3 \%)$ were found in the reference group.

Table 1 Anthropometric, clinical and metabolic characteristics of subjects enrolled in the study ${ }^{\mathrm{a}}$

\begin{tabular}{llll}
\hline $\begin{array}{l}\text { Anthropometric, clinical } \\
\text { and metabolic characteristics }\end{array}$ & Reference group $(n=33)$ & Hypertensive group $(n=55)$ & $p$ \\
\hline Age (years) & $16(12-18)$ & $17(11-18)$ & NS \\
BMI $Z$-score & $-0.03(-1.62$ to 3.83$)$ & $2.12(-1.11$ to 9.48$)$ & $<0.01$ \\
Creatinine $(\mathrm{mg} / \mathrm{dL})$ & $0.63(0.37-0.89)$ & $0.84(0.48-1.13)$ & $<0.01$ \\
Uric acid $(\mathrm{mg} / \mathrm{dL})$ & $4.6(2.19-5.48)$ & $6.49(3.26-8.89)$ & $<2(12-57)$ \\
Urea $(\mathrm{mg} / \mathrm{dL})$ & $22(14-35)$ & $104(32-238)$ & $<0.01$ \\
Triglycerides $(\mathrm{mg} / \mathrm{dL})$ & $78(56-134)$ & $1.44(0.44-18.87)$ & $<0.05$ \\
Urine creatinine $(\mathrm{mg} / \mathrm{dL})$ & $0.91(0.21-2.06)$ & $145.6(113.9-233.3)$ & $<0.05$ \\
eGFR $\left(\mathrm{ml} / \mathrm{min} / 1.73 \mathrm{~m}^{2}\right)$ & $154.4(103.9-265.26)$ & $25.15(7-75.14)$ & $<0.01$ \\
Renin activity $(\mathrm{pg} / \mathrm{mL})$ & $18.45(7.24-38.34)$ & $3,790.9(238.8-9361.9)$ & $<0.05$ \\
Aldosterone $(\mathrm{pg} / \mathrm{mL})$ & $2,872.7(1,032.3-5972.2)$ & $0.17(0-2.28)$ & $<0.01$ \\
AGT/cr. $(\mathrm{ng} / \mathrm{mg} \mathrm{cr})$ & $0(0-0.44)$ & $<0.01$
\end{tabular}

BMI, Body mass index; eGFR, estimated glomerular filtration rate; AGT, angiotensin; cr., creatinine; NS, not significant

Data are presented as the median with the $95 \%$ confidence interval $(\mathrm{CI})$ in parenthesis

${ }^{a}$ Adolescents enrolled in the study were divided into two groups based on ambulatory blood pressure monitoring (ABPM): a hypertensive (HT) group consisting of patients with confirmed primary HT and a reference $\left.{ }^{\circledR}\right)$ group consisting of patients with white-coat hypertension 
Table 2 Ambulatory blood pressure data

D, day; N, night; L, load; SBD, systolic blood pressure; DBP, diastolic blood pressure; $\mathrm{R}$, reference group; HT, hypertensive group (examined group)

Data are presented as the median with the $5 \% \mathrm{CI}$ in parenthesis

\begin{tabular}{llll}
\hline ABPM parameters & Reference group & Hypertensive group & $p$ \\
\hline Mean SBP/24 h (mmHg) & $116(105-126)$ & $132(115-156)$ & $<0.01$ \\
Mean DBP/24 h (mmHg) & $66(54-77)$ & $72(58-93)$ & $<0.01$ \\
LSBP (\%) D & $19(0-25)$ & $67.2(3,2-100)$ & $<0.01$ \\
LSBP (\%) N & $14(0-20)$ & $33.3(0-100)$ & $<0.01$ \\
LDBP (\%) D & $6.6(0-25)$ & $16.2(0-85,7)$ & $<0.05$ \\
LDBP (\%) N & $5.9(0-20)$ & $7.8(0-100)$ & NS \\
\hline
\end{tabular}

The body height, weight and BMI of HT adolescents were higher compared to those in the reference group ( $p<$ 0.01 ). The median BMI $Z$-score in the HT group was 2.12 (range -1.11 to 9.48 ) and was higher than the median of the reference group $(p<0.01)$. Thirty-seven subjects $(67.3 \%)$ from the HT group were classified as overweight or obese.

From the data in Fig. 1, it is apparent that the AGT/cr. ratio was significantly increased in the subjects in the HT group in comparison to the healthy controls. Similar significant differences were found in plasma renin activity, plasma aldosterone level, SUA level, creatinine, urea, triglycerides and eGFR between the HT subjects and the healthy reference subjects.

The median SUA concentration in HT subjects was significantly higher than that in the subjects of the reference group [6.49 (3.26-8.89) vs. $4.6(2.19-5.48) \mathrm{mg} / \mathrm{dL}$, respectively; $p<0.01]$. The SUA level exceeded $5.5 \mathrm{mg} / \mathrm{dL}$ in $78.2 \%$ of the subjects with HT.

We performed single regression and correlation analyses of the AGT/cr. ratio according to clinical parameters (Fig. 2) and found a significant nonlinear correlation between the urine AGT/cr. ratio and SUA level $(r=0.508, p<0.01)$. Additionally, the urine AGT/cr. ratio was positively correlated with height ( $r=0.32, p<0.01)$, body weight $(r=0.34, p<0.01)$, BMI $Z$-score $(r=0.24, p<0.05)$ and triglyceride levels $(r=$

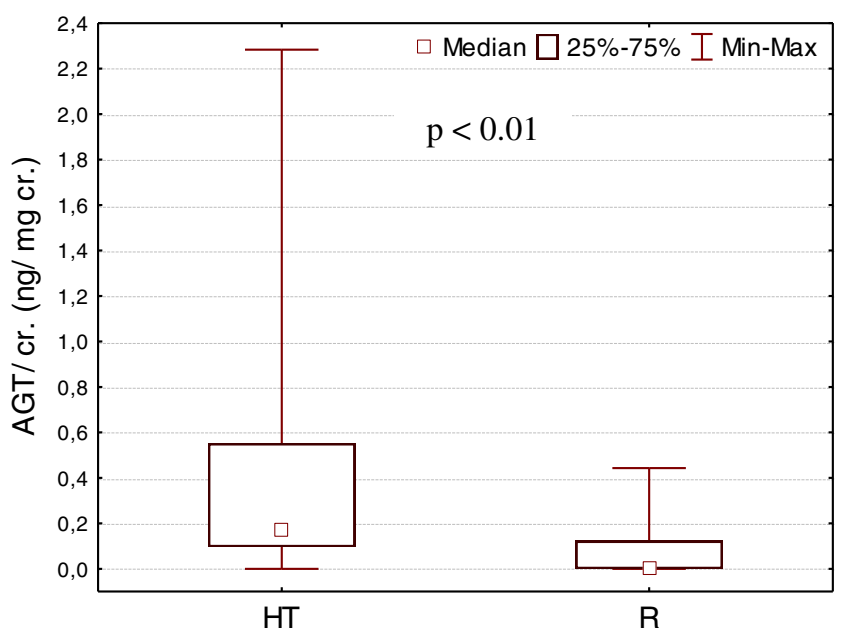

Fig. 1 Comparison of angiotensin/creatinine $(A G T / c r$. $)$ ratio between the hypertensive (HT) subjects with confirmed primary HT and the reference $(R)$ subjects with white-coat hypertension
$0.29, p<0.05$ ). In a subsequent analysis we checked the relationships between the AGT/cr. ratio and plasma renin activity and plasma aldosterone level. In the subjects in the HT group, the AGT/cr. ratio correlated positively with plasma aldosterone level $(r=0.40, p<0.01)$ and plasma renin activity $(r=0.30, p<0.05)$; in contrast, in the reference group we did not find any statistically significant correlation between these parameters.

Because of the known effects of gender, age and parameters of physical development differences on SUA, we performed multivariable linear regression to control for gender, age, BMI $Z$-score and serum creatinine, triglyceride and cholesterol levels. We selected statistically significant explanatory variables such that the sum of squared residuals would be minimal in the model. As a result gender, BMI $Z$ score and serum creatinine level were excluded $(p=0.68, p=$ $0.65, p=0.71$, respectively). The model with the remaining four parameters (SUA, triglycerides, cholesterol and age) accounted for more than $41 \%$ of the variations in the AGT/cr. ratio $\left(r^{2}=0.412, p<0.001\right.$ for the model).

The results of the regression analysis of the AGT/cr. ratio and SUA are summarized in Table 3.

The relationship between AGT/cr. levels and SUA levels after controlling for age, gender and BMI $Z$-score continued

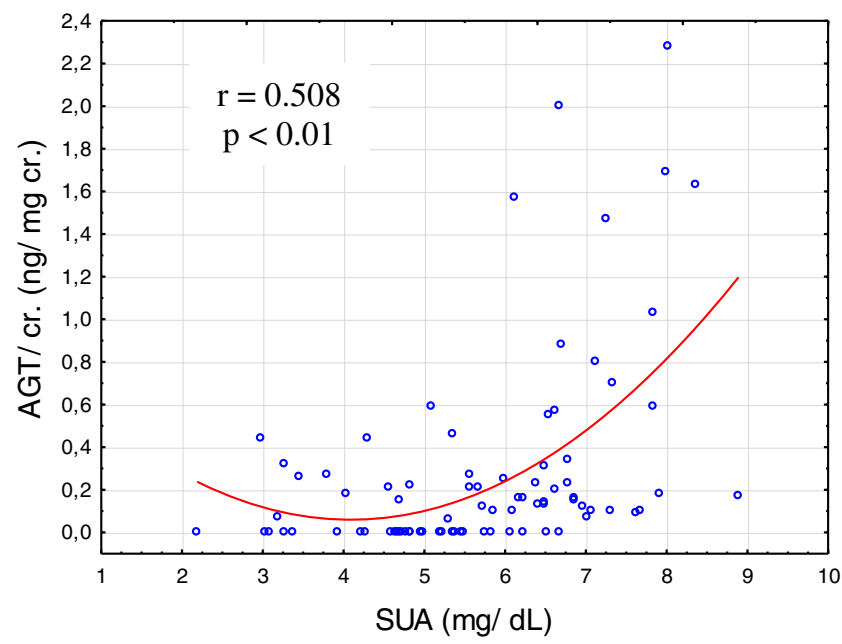

Fig. 2 Exponential regression analysis demonstrating the relationship between the angiotensin/creatinine $(A G T / c r)$. ratio and serum creatinine, urea and uric acid $(S U A)$ in all subjects 
Table 3 Multivariate regression analysis of angiotensin/creatinine (AGT/cr). ratio and serum uric acid

\begin{tabular}{lllc}
\hline Clinical parameters & Standardized $\beta$ & Standard error & $p$ \\
\hline Uric acid (mg/dL) & 0.204 & 0.139 & 0.04 \\
Triglycerides (mg/ dL) & 0.413 & 0.170 & 0.02 \\
Cholesterol (mg/dL) & -0.632 & 0.162 & $<0.001$ \\
Age (years) & -0.344 & 0.129 & 0.01 \\
\hline
\end{tabular}

to show a significant association. Based on the 24-h ABPM results, all ABPM parameters, except for nighttime DBP load, were significantly higher in adolescents with primary HT than in those in the reference group.

We also analyzed the relationship between AGT/cr. and the parameters derived from ABPM. We found a positive correlation between AGT/cr. and mean SBP/24 h $(r=0.24, p<0.05)$, AGT/cr. and mean SBP during the daytime $(r=0.27, p<0.05)$ and AGT/cr. and daytime SBP loads $(r=0.31, p<0.05)$.

Further statistical tests revealed a significant difference between urine AGT levels in subjects with HT and hyperuricemia $[\mathrm{HU}(+)]$ and subjects with $\mathrm{HT}$ and normouricemia $[\mathrm{HU}(-)$; median $0.2(0.0-3.27)$ vs. $0.13(0.0-0.3)$, respectively; $p=$ $0.002)]$. However, the significance was lost after the correction for creatinine. This result may possibly be explained by the lower number of subjects in the normouricemia group.

In the $\mathrm{HU}(+)$ group the $\mathrm{AGT} / \mathrm{cr}$. ratio was positively correlated with plasma renin activity $(r=0.35, p<0.05)$ and plasma aldosterone level $(r=0.39, p<0.05)$.

\section{Discussion}

In this cross-sectional study, the AGT/cr. ratio was significantly higher in hypertensive adolescents than in teenagers in whom diagnosis of HT was not confirmed in 24-h ABPM (reference group - white-coat HT). Other important findings were positive correlations between AGT/cr. and height, body weight, BMI, SUA and triglyceride levels. It is interesting to note that the relationship between AGT/cr. levels and SUA levels after controlling for age, gender and BMI Zscore continued to a show significant association. The multivariable linear regression model that included SUA, triglycerides, cholesterol and age accounted for more than $41 \%$ of the variations in the $\mathrm{AGT} / \mathrm{cr}$. ratio.

To the best of our knowledge this is the first clinical study of the association between urinary AGT level and hyperuricemia in children and adolescents.

The prevalence of HT is increasing among young people worldwide. One of the important metabolic abnormalities in children and adolescents with primary HT appears to be elevated SUA levels. Many large epidemiologic studies have demonstrated that an elevated SUA level is associated with HT [4, 23-26]. However, controversy still remains on whether uric acid is an independent predictor, a mediator or just a marker of the development of HT. The increased uric acid level has been reported to induce HT by activating the RAS and up-regulating proinflammatory mediators in VSMCs $[27,28]$. A study carried out in a large, nationally representative sample of 6,036 U.S. adolescents (age range 12-17 years) has shown that SUA is associated with elevated BP [29]. Furthermore, participants with a uric acid level of $\geq 5.5 \mathrm{mg} / \mathrm{dL}$ had a twofold higher odds ratio of having elevated BP compared to those with a uric acid level of $\leq 5.5 \mathrm{mg} / \mathrm{dL}$.

Corry et al. [30] reported that uric acid stimulated the major components of the vascular RAS, including the stimulation of both AGT and angiotensin II production. Recent attention has focused on findings that the proximal intratubular concentrations of angiotensin I and II are much greater than their corresponding plasma concentrations, which emphasizes the role of the local/tissue RAS in specific tissues $[31,32]$. Also, the finding that there is a very high formation of angiotensin I following the incubation of proximal tubular fluid with excessive renin indicates a very high AGT substrate availability in this segment [31,33].

Kobori et al. $[17,18]$ reported that urinary AGT excretion provides a specific index of the intrarenal RAS status in angiotensin II-dependent hypertensive rats. They also showed that the enhanced production of urinary AGT is not a nonspecific consequence of proteinuria. The results of another study demonstrated that the infusion of human AGT into hypertensive and normotensive rats did not result in its presence in the urine, suggesting that circulating AGT is not a source of urinary AGT [18]. van den Heuvel et al. suggested that the urinary AGT level reflects both renal dysfunction and the beneficial effects of the RAS blockade [34]. Contrary to expectations, in our study we found a statistically significant association between the urine AGT/cr. ratio and systemic activation of RAS in hypertensive patients; however, we only measured plasma aldosterone and plasma renin activity, but not plasma AGT. Our results may be supported by those of Kobori et al. [19] who showed a significant reduction of the AGT/cr. ratio in patients with HT treated with RAS blockers. These data suggest that the efficacy of the RAS blockade to reduce the intrarenal RAS activity can be assessed by measurements of urinary AGT excretion [19]. In the reference group we did not find statistically significant correlations between these parameters.

Yamamoto et al. reported a lack of significant correlation between urinary and plasma AGT levels [35].

It is plausible to consider that plasma AGT cannot easily filter across the glomerular membrane because of its size [12]. In light of this fact it is unlikely that most of the increased urinary AGT in hypertensive patients with slight 
proteinuria originates from the circulation. However, the possible contribution of derangement of glomerular permeability, which could result from progressive renal fibrosis due to the increased urinary AGT level, cannot be ruled out.

Previous methods for determining the concentration of AGT in the urine were difficult to perform and prone to generate significant methodological errors. In 2007, Katsurada et al. developed a sandwich ELISA system to directly and sensitively measure urinary human AGT [16].

There are limited data on AGT excretion in the literature. Kobori et al. described similar AGT excretion values as those reported by us in patients with chronic kidney disease [36]. The AGT/cr. ratio in patients with type 2 diabetic nephropathy or with membranous nephropathy were much higher than the overall average levels in patients with chronic kidney disease [36]. Importantly, Mezzano et al. reported that the activation of the intrarenal RAS participates in the progression of renal injury in patients with diabetic nephropathy [37] and membranous nephropathy [38]. In contrast, Kobori et al. [36] demonstrated the AGT/cr. levels in their patients with minimal change disease were similar to those in the control group, even though the patients with minimal change had severe proteinuria. The above data and also the results from a number animal studies on HT clearly indicate that augmented AGT excretion is not simply a nonspecific consequence of proteinuria. In our study, we found no correlation between urinary AGT concentration and albuminuria levels. Similar observations have been reported by Lantelme et al. [39] in adult patients with HT.

In our study the urinary excretion of AGT correlated with some components of metabolic syndrome, such as obesity, hyperuricemia and serum triglyceride levels.

The statistical analysis included multivariate analysis with the presentation of the independent factors associated with an increased urine AGT/cr. ratio. The multivariable linear regression model excluded the influence of gender, BMI Z-score and serum creatinine level, but included the parameter triglycerides, which might have a positive impact on the urine AGT/cr. ratio. The impact of serum cholesterol was significant, but negative, so should not be considered. However, more research on this topic needs to be undertaken before the association between the AGT/cr. ratio and metabolic syndrome is more clearly understood.

In summary, although our investigations into this area are still ongoing, our preliminary results allow us to draw the following conclusions: (1) children and adolescents with primary HT have an increased urinary excretion of AGT; (2) hyperuricemia appears to be a factor which significantly increases the excretion of AGT in adolescents with primary HT, although large, multicenter studies are needed to confirm this observation. Finally, a number of important limitations need to be considered. First, the reference group was not a group of healthy teenagers, but a group of patients in whom hypertension was not confirmed in 24-h ABPM; consequently these subjects are considered to belong to the white-coat HT group. Secondly, the two study groups clearly differed: the hypertensive group consisted of adolescent males mainly with a high BMI $Z$-score and the reference group included slim females with white-coat HT. However, as the multivariate analysis was able to show that neither gender nor the BMI $Z$-score influenced the relationship between the AGT/cr. ratio and SUA levels.

However, despite the limitations of this study, we believe that the unique position of AGT in the RAS and its function underlines the importance of this protein as attractive target in developing new effective strategies for the management of many diseases, particularly HT, atherosclerosis and obesity. At present, there is still no information in the literature on the role of AGT produced in local tissues in the pathophysiology of HT, nor on the correlation of AGT produced in situ with the circulating pool of AGT.

Acknowledgments This study was supported by a research grant from the Medical University of Bialystok (No 123-41765L).

\section{Conflict of interest None.}

Open Access This article is distributed under the terms of the Creative Commons Attribution License which permits any use, distribution, and reproduction in any medium, provided the original author(s) and the source are credited.

\section{References}

1. Masuo K, Kawaguchi H, Mikami H, Ogihara T, Tuck ML (2003) Serum uric acid and plasma norepinephrine concentrations predict subsequent weight gain and blood pressure elevation. Hypertension $42: 474-480$

2. Sundström J, Sullivan L, D’Agostino RB, Levy D, Kannel WB, Vasan RS (2005) Relations of serum uric acid to longitudinal blood pressure tracking and hypertension incidence. Hypertension 45:28-33

3. Mazzali M, Hughes J, Kim YG, Jefferson JA, Kang DH, Gordon KL, Lan HY, Kivlighn S, Johnson RJ (2001) Elevated uric acid increases blood pressure in the rat by a novel crystal-independent mechanism. Hypertension 38:1101-1106

4. Perlstein TS, Gumieniak O, Williams GH, Sparrow D, Vokonas PS, Gaziano M, Weiss ST, Litonjua AA (2006) Uric acid and the development of hypertension: the normative aging study. Hypertension 48:1031-1036

5. Feig DI, Johnson RJ (2003) Hyperuricemia in childhood primary hypertension. Hypertension 42:247-252

6. Saito I, Saruta T, Kondo K, Nakamura R, Oguro T, Yamagami K, Ozawa Y, Kato E (1978) Serum uric acid and the renin-angiotensin system in hypertension. J Am Geriatr Soc 26:241-247

7. Dzau VJ, Re R (1994) Tissue angiotensin system in cardiovascular medicine. A paradigm shift? Circulation 89:493-498

8. Baltatu O, Silva JA Jr, Ganten D, Bader M (2000) The brain reninangiotensin system modulates angiotensin II-induced hypertension and cardiac hypertrophy. Hypertension 35:409-412

9. Dell'Italia LJ, Meng QC, Balcells E, Wei CC, Palmer R, Hageman GR, Durand J, Hankes GH, Oparil S (1997) Compartmentalization of angiotensin II generation in the dog heart evidence for 
independent mechanisms in intravascular and interstitial spaces. $\mathrm{J}$ Clin Invest 100:253-258

10. Mazzocchi G, Malendowicz LK, Markowska A, Albertin G, Nussdorfer GG (2000) Role of adrenal renin-angiotensin system in the control of aldosterone secretion in sodium-restricted rats. Am J Physiol Endocrinol Metab 278:E1027-E1030

11. Danser AH, Admiraal PJ, Derkx FH, Schalekamp MA (1998) Angiotensin I-to-II conversion in the human renal vascular bed. J Hypertens 16:2051-2056

12. Navar LG, Harrison-Bernard LM, Nishiyama A, Kobori H (2002) Regulation of intrarenal angiotensin II in hypertension. Hypertension 39:316-322

13. Kobori H, Nangaku M, Navar LG, Nishiyama A (2007) The intrarenal renin-angiotensin system: from physiology to the pathobiology of hypertension and kidney disease. Pharmacol Rev 59:251-287

14. Kobori H, Ozawa Y, Suzaki Y, Prieto-Carrasquero MC, Nishiyama A, Shoji T, Cohen EP, Navar LG (2006) Young Scholars Award Lecture: intratubular angiotensinogen in hypertension and kidney diseases. Am J Hypertens 19:541-550

15. Kobori H, Ozawa Y, Satou R, Katsurada A, Miyata K, Ohashi N, Hase N, Suzaki Y, Sigmund CD, Navar LG (2007) Kidney-specific enhancement of ANG II stimulates endogenous intrarenal angiotensinogen in gene-targeted mice. Am J Physiol Renal Physiol 293:F938-F945

16. Katsurada A, Hagiwara Y, Miyashita K, Satou R, Miyata K, Ohashi N, Navar LG, Kobori H (2007) Novel sandwich ELISA for human angiotensinogen. Am J Physiol Renal Physiol 293: F956-F960

17. Kobori H, Harrison-Bernard LM, Navar LG (2002) Urinary excretion of angiotensinogen reflects intrarenal angiotensinogen production. Kidney Int 61:579-585

18. Kobori H, Nishiyama A, Harrison-Bernard LM, Navar LG (2003) Urinary angiotensinogen as an indicator of intrarenal Angiotensin status in hypertension. Hypertension 41:42-49

19. Kobori H, Alper AB Jr, Shenava R, Katsurada A, Saito T, Ohashi N, Urushihara M, Miyata K, Satou R, Hamm LL, Navar LG (2009) Urinary angiotensinogen as a novel biomarker of the intrarenal reninangiotensin system status in hypertensive patients. Hypertension 53:344-350

20. Urbina E, Alpert B, Flynn J, Hayman L, Harshfield GA, Jacobson M, Mahoney L, McCrindle B, Mietus-Snyder M, Steinberger J, Daniels S, American Heart Association Atherosclerosis, Hypertension, and Obesity in Youth Committee (2008) Ambulatory blood pressure monitoring in children and adolescents: recommendations for standard assessment: a scientific statement from the American Heart Association Atherosclerosis, Hypertension, and Obesity in Youth Committee of the council on cardiovascular disease in the young and the council for high blood pressure research. Hypertension $52: 433-451$

21. Wühl E, Witte K, Soergel M, Mehls O, Schaefer F, German Working Group on Pediatric Hypertension (2002) Distribution of 24-h ambulatory blood pressure in children: normalized reference values and role of body dimensions. J Hypertens 20:1995-2007

22. Yoon EY, Cohn L, Rocchini A, Kershaw D, Freed G, Ascione F, Clark S (2012) Antihypertensive prescribing patterns for adolescents with primary hypertension. Pediatrics 129:e1-e8

23. Feig DI, Kang DH, Johnson RJ (2008) Uric acid and cardiovascular risk. N Engl J Med 359:1811-1821
24. Feig DI, Kang DH, Nakagawa T, Mazzali M, Johnson RJ (2006) Uric acid and hypertension. Curr Hypertens Rep 8:111-115

25. Mellen PB, Bleyer AJ, Erlinger TP, Evans GW, Nieto FJ, Wagenknecht LE, Wofford MR, Herrington DM (2006) Serum uric acid predicts incident hypertension in a biethnic cohort. Hypertension 48:1037-1042

26. Selby JV, Friedman GD, Quesenberry CP (1990) Precursors of essential hypertension: pulmonary function, heart rate, uric acid, serum cholesterol, and other serum chemistries. Am J Epidemiol 131:1017-1027

27. Johnson RJ, Kang DH, Feig D, Kivlighn S, Kanellis J, Watanabe S, Tuttle KR, Rodriguez-Iturbe B, Herrera-Acosta J, Mazzali M (2003) Is there a pathogenetic role for uric acid in hypertension and cardiovascular and renal disease? Hypertension 41:1183-1190

28. Leyva F, Anker SD, Godsland IF, Teixeira M, Hellewell PG, Kox WJ, Poole-Wilson PA, Coats AJ (1998) Uric acid in chronic heart failure: a marker of chronic inflammation. Eur Heart J 19:1814-1822

29. Loeffler LF, Navas-Acien A, Brady TM, Miller ER 3rd, Fadrowski JJ (2012) Uric acid level and elevated blood pressure in US adolescents: National Health and Nutrition Examination Survey, 1999-2006. Hypertension 59:811-817

30. Corry DB, Eslami P, Yamamoto K, Nyby MD, Makino H, Tuck ML (2008) Uric acid stimulates vascular smooth muscle cell proliferation and oxidative stress via the vascular renin-angiotensin system. J Hypertens 26:269-275

31. Navar LG, Harrison-Bernard LM, Wang CT, Cervenka L, Mitchell KD (1999) Concentrations and actions of intraluminal angiotensin II. J Am Soc Nephrol 10:S189-S195

32. Seikaly MG, Arant BS Jr, Seney FD Jr (1990) Endogenous angiotensin concentrations in specific intrarenal fluid compartments of the rat. J Clin Invest 86:1352-1357

33. Navar LG, Imig JD, Zou L, Wang CT (1997) Intrarenal production of angiotensin II. Semin Nephrol 17:412-422

34. van den Heuvel M, Batenburg WW, Jainandunsing S, Garrelds IM, van Gool JM, Feelders RA, van den Meiracker AH, Danser AH (2011) Urinary renin, but not angiotensinogen or aldosterone, reflects the renal renin-angiotensin-aldosterone system activity and the efficacy of renin-angiotensin-aldosterone system blockade in the kidney. J Hypertens 29:2147-2155

35. Yamamoto T, Nakagawa T, Suzuki H, Ohashi N, Fukasawa H, Fujigaki Y, Kato A, Nakamura Y, Suzuki F, Hishida A (2007) Urinary angiotensinogen as a marker of intrarenal angiotensin II activity associated with deterioration of renal function in patients with chronic kidney disease. J Am Soc Nephrol 18:1558-1565

36. Kobori H, Ohashi N, Katsurada A, Miyata K, Satou R, Saito T, Yamamoto T (2008) Urinary angiotensinogen as a potential biomarker of severity of chronic kidney diseases. J Am Soc Hypertens 2:349-354

37. Mezzano S, Droguett A, Burgos ME, Ardiles LG, Flores CA, Aros CA, Caorsi I, Vio CP, Ruiz-Ortega M, Egido J (2003) Reninangiotensin system activation and interstitial inflammation in human diabetic nephropathy. Kidney Int 64:S64-S70

38. Mezzano SA, Aros CA, Droguett A, Burgos ME, Ardiles LG, Flores CA, Carpio D, Vio CP, Ruiz-Ortega M, Egido J (2003) Renal angiotensin II up-regulation and myofibroblast activation in human membranous nephropathy. Kidney Int 64:S39-S45

39. Lantelme P, Rohrwasser A, Vincent M, Cheng T, Gardier S, Legedz L, Bricca G, Lalouel JM, Milon H (2005) Significance of urinary angiotensinogen in essential hypertension as a function of plasma renin and aldosterone status. J Hypertens 23:785-792 